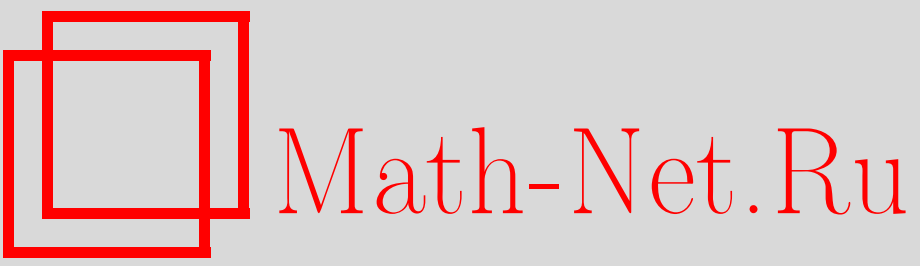

А. Джиакоббе, Выпуклость и многозначные гамильтонианы, УМH, 2000, том 55, выпуск 3, 177-178

DOI: https://doi.org/10.4213/rm300

Использование Общероссийского математического портала Math-Net.Ru подразумевает, что вы прочитали и согласны с пользовательским соглашением

http://www.mathnet.ru/rus/agreement

Параметры загрузки:

IP : 54.157 .27 .8

26 апреля 2023 г., $17: 49: 45$ 


\section{ВЫПУКЛОСТЬ И МНОГОЗНАЧНЫЕ ГАМИЛЬТОНИАНЫ}

\section{А. ДжиАкоБье}

Симплектическое действие связной группы Ли $G$ на симплектическом многообразии $\left(W^{2 N}, \Omega\right)$ определяет отображение $\left(\xi \mapsto X_{\xi}\right)$ алгебры Ли $\mathfrak{g}$ группы $G$ в векторные поля на многообразии, называемое инфинитезимальным действием. Опускание индекса при помощи симплектической 2-формы дает отображение $\left(\xi \mapsto \tau_{\xi}\right)$ алгебры Ли g в замкнутые 1-формы на $W$. Эти 1-формы $\tau_{\xi}$ называются гамильтонианами. Если действие гамильтоново (что означает точность всех гамильтонианов), то можно определить отображение моментов $\mu: W \rightarrow \mathfrak{g}^{*}$.

Если дополнительно $G$ является тором $\mathbf{T}$, то образ такого отображения является выпуклым многогранником, как это было показано в [1] и [2].

Когда не все гамильтонианы точны, возможно определить обобщенное отображение моментов. Это отображение определено на минимальной абелевой накрывающей симплектического многообразия (на которой все гамильтонианы поднимаются до точных 1-форм). В случае тора T образ этого отображения является выпуклым неограниченным многогранником, так как содержит целое подпространство направлений многозначности. Развитая техника позволяет получить некоторые другие интересные факты: гамильтоновы действия являются устойчивыми относительно возмущений симплектической формы, а действие тора (с неподвижными точками) размерности вдвое меншше размерности симплектического многообразия является обязательно гамильтоновым.

Условие однозначности гамильтонианов не подходит для некоторых интересных физических ситуаций, как показано в [3]. Вопрос справедливости теоремы выпуклости при ослаблении условия однозначности гамильтонианов является интересным как в физике, так и в математике.

Для замкнутых 1-форм $\tau_{\vartheta}, \vartheta \in \mathfrak{t}$, где $\mathfrak{t}$ - алгебра Ли тора $\mathbf{T}$, все критические многообразия являются симплектическими (и, следовательно, четномерными); более того, индекс $\tau_{\vartheta}$ на критическом многообразии является четным, а следовательно, таковым является и коиндекс. Это следует из локального выражения для отображения моментов. Индекс $\tau_{\vartheta}$ в критической точке есть удвоенное число весов $\alpha_{j}$ таких, что $\left\langle\alpha_{j}, \vartheta\right\rangle<0$.

Теорема 1. Пусть $\tau$-замкнутая 1-форма Морса-Ботта без критических подмногообразий индекса 1 на компактном многообразии $W$. Если у т есть локальный минимум, то он единственный, а $\tau$ - точная форма.

Предложение может быть доказано сначала для рациональных 1-форм. Рациональная 1-форма корректно определяет $S^{1}$-значное отображение. Это отображение может быть пропущено через граф, вершпны которого соответствуют критическим многообразиям данной 1-формы. Стандартные комбинаторные рассуждения на графе завершают доказательство для рациональных 1-форм. Для произвольных 1-форм теорема верна потому, что любая замкнутая 1-форма Морса-Ботта степени иррациональности $r(r>1)$ может быть аппроксимирована замкнутой 1-формой Морса-Ботта с теми жее особенностями и степенъю иррациональности $r-1$.

Первым следствием этой теоремы является

Теорема 2. Если действие тора $\mathbf{T}^{n}$ на компактном симплектическом многообразии $\left(W^{2 n}, \Omega\right)$ имеет неподвижные точки, то действие гамильтоново.

ДокАЗАтЕльство. Предположим, что действие является эффективным. Рассмотрим неподвижную точку $p$. Ковекторы $\alpha_{j}$ должны порождать (над $\left.\mathbb{Z}\right) \mathbb{Z}^{n}$ и их не может быть больше $n$. Следовательно, после $S L(n, \mathbb{Z})$-замены координат в $\mathrm{t}^{*}$ получаем, что $\alpha_{j}=e_{j}, e_{1}=(1,0, \ldots, 0)$, $\ldots, e_{n}=(0, \ldots, 0,1)$. Теперь выберем произвольный $\vartheta \in \mathfrak{t}$ из первого октанта, т.е. $\vartheta$ с положительными координатами относительно канонического базиса. 1-форма $\tau_{\vartheta}$ имеет локальный минимум в $p$ и, следовательно, должна быть точной. В этом случае подпространство $\mathfrak{t}_{e}$ векторов в $\mathfrak{t}$, соответствующих точным гамильтонианам, совпадает с алгеброй Ли $\mathfrak{t}$. Отсюда следует гамильтоновость действия. 
Теорема 3. На компактном симплектическом многообразии с симплектическим действием тора подпространство $\mathfrak{t}_{e}$ векторов из $\mathfrak{t}$, соответствующих точным гамильтонианам, является рациональным подпространством. Это подпространство не меняется при мальх возмущениях симплектической формы.

ДокАзАТЕльство. Нужно показать: если 1-форма $\tau_{\vartheta}$ точна (для каждого вектора $\vartheta \in \mathfrak{t}$ ), то она должна остаться точной при малой деформации симплектической формы. Аналогично для векторов $\vartheta \in \mathfrak{t}$, соответствующих неточным формам $\tau_{\vartheta}$.

Имеется одно интуитивно понятное следствие этой теоремы: многозначные гамилтонианы остаются многозначными. Для неточной $\tau_{\vartheta}$ верно, что $\int_{\gamma} \tau_{\vartheta} \neq 0$ для некоторой нетривиальной петли $\gamma$ из фундаментальной группы многообразия. При любой малой деформации симплектической формы $\Omega$ 1-форма $\tau_{\vartheta}$ меняется гладким образом, и, соответственно, гладко меняется ее интеграл, которьй должен оставаться ненулевым при малых деф̆ормациях $\Omega$ (неточность является устойчивым свойством).

Что остается неясным, так это локальная устойчивость точных гамильтонианов. Ранее мы доказали, что $\tau_{\vartheta}$ является точной тогда и только тогда, когда у нее есть минимум, что эквивалентно существованию точки $p$, неподвижной для $\tau_{\vartheta}$.

Но самом деле от симплектической структуры $\Omega$ зависит только знак этих весов, причем зависит непрерывным образом, следовательно, веса являются устойчивыми относительно малых возмущений $\Omega$. Соответственно, существование или отсутствие неподвижных точек локально не зависит от $\Omega$, а значит, таким же является и свойство точности/неточности формы.

Это приводит к другой теореме, которая является естественным обобщением результатов из [1], [2]:

Теорема 4. Пусть тор $\mathbf{T}^{n}$ действует на симплектическом многообразии $\left(W^{2 N}, \Omega\right)$. Существует абелево накрытие $\widetilde{W}$, на котором каждая 1-форма $\tau_{\vartheta}$ поднимается до точной 1-формы. $\widetilde{W}$ есть минимальное накрытие, на котором отображение моментов определено как функция. Образ отображения моментов $\widetilde{\mu}: \widetilde{W} \rightarrow \mathfrak{t}^{*}$ является выпуклым, но возможсн некомпактным многогранником.

В самом деле, образ $\widetilde{\mu}(\widetilde{W})$ получается следующим образом: рассмотрим максимальный тор $\mathbf{T}_{e}$, отвечающий однозначным гамильтонианам. Его неподвижные многообразия $Z_{j}(j$ пробегает конечное множество) определяют вершины выпуклого ограниченного многогранника $P$ в $\mathfrak{t}_{e}^{*}$, которьй является образом ограничения отображения моментов на $\mathbf{T}_{e}$. Множество $\widetilde{\mu}(\widetilde{W})$ является выпуклым многогранником $P \times\left(\mathfrak{t}_{e}^{*}\right)^{\perp}$.

Можно доказать утверждение, параллельное результату в [4]. В этом случае выпуклый многогранник является пересечением образа многозначного отображения моментов с некоторой камерой Вейля.

ТеОРема 5. Пусть $W$ - симплектическое многообразие с симплектическим действием компактной группь Ли $G$. Тогда существует отображение моментов, определенное на соответствующей накрывающей многообразия $W$ со значениями в сопряженном пространстве к алгебре Ли группь $G: \widetilde{\mu}: \widetilde{W} \rightarrow \mathfrak{g}^{*}$.

Пересечение образа $\widetilde{\mu}$ с положительной камерой Вейля является выпуклым многогранником.

\section{СПИСОК ЛИТЕРАТУРЫ}

[1] Atiyah M. F. // Bull. London Math. Soc. 1982. V. 14. P. 1-15. [2] Guillemin V., Sternberg S. // Invent. Math. 1982. V. 67. P. 491-513. [3] Новиков С. П. // УМН. 1982. Т. 37. № 5. C. 3-49. [4] Kirwan F. // Invent. Math. 1984. V. 77. P. 547-552.

University of Maryland, College Park

Принято редколлегией 03.04 .2000 